\title{
Fractional monodromy in systems with coupled angular momenta
}

\author{
M S Hansen ${ }^{1}$, F Faure $^{2}$ and B I Zhilinskii ${ }^{3}$ \\ ${ }^{1}$ Institute of Mathematics, Technical University of Denmark, 2800 Kgs Lyngby, Denmark \\ ${ }^{2}$ Institut Fourier, Université Joseph Fourier, BP 74, 38402 Saint-Martin d'Hères, Cedex, France \\ ${ }^{3}$ Université du Littoral, UMR du CNRS 8101, 59140 Dunkerque, France \\ E-mail: M.S.Hansen@mat.dtu.dk, frederic.faure@ujf-grenoble.fr and zhilin@univ-littoral.fr
}

Received 27 February 2007, in final form 12 September 2007

Published 9 October 2007

Online at stacks.iop.org/JPhysA/40/13075

\begin{abstract}
We present a one-parameter family of systems with fractional monodromy, which arises from a 1:2 diagonal action of a dynamical symmetry $S O(2)$. In a regime of adiabatic separation of slow and fast motions, we relate the presence of fractional monodromy to a redistribution of states both in the quantum and in the semi-quantum spectra.
\end{abstract}

PACS numbers: $03.65 . S q, 02.40 . Y y$

(Some figures in this article are in colour only in the electronic version)

\section{Introduction}

In this paper we consider a simple one-parameter family of Hamiltonian which is a slight generalization of the well-known example of spin-orbit coupling. This latter model has been the object of several studies [1, 6-8], demonstrating the presence of integer monodromy for some interval of parameter values.

We remind here that Hamiltonian monodromy is a generic property of classical integrable systems, intensively studied and popularized by Cushman [5] and described in detail by Duistermaat in 1980 [9]. In a classical dynamical system with two degrees of freedom, the Hamiltonian monodromy can typically appear in one-parameter families through the Hamiltonian Hopf bifurcation [10]. It was shown later that there is a correspondence between the appearance of monodromy within a one-parameter family of classical Hamiltonians and the redistribution of bands in the spectrum of the associated quantum problem [6, 11]. The appearance of Hamiltonian monodromy in the classical system also indicates the presence of a topological bifurcation in a semi-quantum description [12].

Our model has fractional monodromy which is a recent generalization of the integer monodromy concept [13-16]. This is the first example of a system with this property on a compact phase space. 
If we suppose that one angular momentum is much larger than the other, then the first one has a slower motion compared to the second one. One gets a slow-fast dynamic that can be analyzed using a semi-quantum description (the fast motion is quantum while the slow motion is classical). This gives line bundles over the 'slow' classical phase space, whose topology is characterized by Chern indices. These line bundles have a nice interpretation for the exact quantum spectrum: the energy levels form different groups named bands. Each band is associated with a line bundle, and the exact number of levels in each band is given from the Chern index of the bundle using an index formula. These topological phenomena have already been observed and analyzed in other situations [7, 28]. Here, we show how the presence of fractional monodromy is related to the locus of topological bifurcations of the bundles, which imply a change of the Chern indices and redistributions of levels between bands.

Compared to the spin-orbit model already studied in $[1,6-8]$ where there is a 1:1 diagonal action of the group $S O(2)$, here the major change is a 1:2 diagonal action of the symmetry group $S O(2)$, and this is responsible for fractional monodromy.

This paper is a part of the ongoing study of global properties of integrable systems on the one side [14, 17-20] (especially in the context of molecular physics [2, 3, 6, 21-25]) and adiabatically coupled systems [7, 26-28] on the other side.

In sections 1-3 we present the model and discuss the fractional monodromy phenomenon in the classical model, using the generalized moment map. In section 4 we study the quantum model and discuss the quantum manifestation of fractional monodromy in the joint spectrum. In section 5 we suppose an adiabatic regime where one angular momentum is much larger than the other, and discuss the topological interpretation of redistributions of levels between bands.

\section{Presentation of model}

\subsection{Hamiltonian with a dynamical 1:2 symmetry action of $S O(2)$}

Very often global properties of the dynamical model under study are due to the symmetry of the physical problem under consideration. Let $\mathbf{N}=\left(N_{x}, N_{y}, N_{z}\right) \in \mathbb{R}^{3}, \mathbf{S}=\left(S_{x}, S_{y}, S_{z}\right) \in \mathbb{R}^{3}$ with fixed $|\mathbf{N}|=\sqrt{N_{x}^{2}+N_{y}^{2}+N_{z}^{2}}$ and $|\mathbf{S}|=\sqrt{S_{x}^{2}+S_{y}^{2}+S_{z}^{2}}$ be two (effective) angular momenta living on $S^{2} \times S^{2}$. The model we study in this paper admits a diagonal 1:2 group action of $S O(2)$ defined by

$S O(2) \times\left(S^{2} \times S^{2}\right) \rightarrow S^{2} \times S^{2}$

$\left(\phi ; N_{+}, N_{-}, N_{z}, S_{+}, S_{-}, S_{z}\right) \mapsto\left(N_{+} \mathrm{e}^{\mathrm{i} \phi}, N_{-} \mathrm{e}^{-\mathrm{i} \phi}, N_{z}, S_{+} \mathrm{e}^{2 \mathrm{i} \phi}, S_{-} \mathrm{e}^{-2 \mathrm{i} \phi}, S_{z}\right)$,

where $N_{ \pm}=N_{x} \pm \mathrm{i} N_{y}, S_{ \pm}=S_{x} \pm \mathrm{i} S_{y}$. The action defined by (1) can be considered as an a priori condition imposed by the physical model. As soon as the group action is given, a generic Hamiltonian can be constructed as a linear combination of polynomials invariant under the group action (1). This leads to a Hamiltonian which has a $S O(2)$ symmetry generated by

$$
J_{z}=2 S_{z}+N_{z}
$$

(i.e. such that $\left[H_{\lambda}, J_{z}\right]=0$ ):

$H_{\lambda}=\frac{1-\lambda}{|\mathbf{S}|} S_{z}+\lambda\left(\frac{1}{|\mathbf{S} \| \mathbf{N}|} S_{z} N_{z}+\frac{1}{2|\mathbf{S} \| \mathbf{N}|^{2}}\left(N_{-}^{2} S_{+}+N_{+}^{2} S_{-}\right)\right), \quad 0 \leqslant \lambda \leqslant 1$.

Here $\lambda$ is a coupling parameter. It can be due to an external magnetic field, for example, recall that the amplitudes $|\mathbf{S}|,|\mathbf{N}|$ are held fixed and in this paper, we only consider the case $|\mathbf{N}|>2|\mathbf{S}|^{4}$.

${ }^{4}$ A preliminary study of the case $|\mathbf{N}|<2|\mathbf{S}|$ has been initiated in [29]. 
The $S O(2)$ symmetry generated by $J_{z}=2 S_{z}+N_{z}$ rotates simultaneously $\mathbf{N}$ and $\mathbf{S}$ about their respective $z$-axes. In [6] the $S O(2)$ action on the phase space $S^{2} \times S^{2}$ was 1:1 diagonal but now the asymmetric appearance of $\mathbf{N}$ and $\mathbf{S}$ implies that while $\mathbf{N}$ is rotated by angle $\phi$, $\mathbf{S}$ is rotated by $2 \phi$.

\subsection{Quantum description and the semi-classical limit}

We begin by a presentation of the quantum system and deduce after the classical one in the semi-classical limit ${ }^{5}$.

Let $\mathbf{N}, \mathbf{S}$ be the angular momentum operators [31] generating an irreducible representation of $\mathfrak{s u}(2) \times \mathfrak{s u}(2)$ in a Hilbert space $\mathcal{H}=\mathcal{H}_{N} \otimes \mathcal{H}_{S}$ of dimension $(2 N+1)(2 S+1) . \quad N, S$ are the respective angular momentum quantum numbers taking integer or half-integer values. Similarly $\hat{H}_{\lambda}$, equation (3), is the Hamiltonian operator. The quantum description is given by the Schrödinger equation (with $\hbar \equiv 1$ )

$$
\mathrm{i} \frac{\mathrm{d} \psi(t)}{\mathrm{d} t}=\hat{H}_{\lambda} \psi(t)
$$

where $\psi(t)$ is a vector in $\mathcal{H}$. To study the semi-classical limit of large quantum numbers $N, S \gg 1$, we introduce the normal symbol of $\hat{H}$ [34]

$$
\left\langle\mathbf{N}, \mathbf{S}\left|\hat{H}_{\lambda}\right| \mathbf{N}, \mathbf{S}\right\rangle=H_{\lambda}+\mathcal{O}\left(\hbar_{N, S}\right),
$$

which is a power series in $\hbar_{N}=1 /(2 N), \hbar_{S}=1 /(2 S)$. Here $|\mathbf{N}, \mathbf{S}\rangle$ are $S U(2)$ coherent states often used to study the semi-classical limit of angular momentum dynamics.

Keeping only the first term of (5) we have a classical Hamiltonian, $H_{\lambda}$, which is the principal symbol of $\hat{H}_{\lambda}$. The dynamics is approximately described by classical angular momenta $\mathbf{N}, \mathbf{S}$ moving according to Hamilton's equations of motion [4]:

$$
\frac{\mathrm{d}}{\mathrm{d} t} \mathbf{N}=\hbar_{N} \partial_{\mathbf{N}} H_{\lambda} \wedge \mathbf{N}, \quad \frac{\mathrm{d}}{\mathrm{d} t} \mathbf{S}=\hbar_{S} \partial_{\mathbf{S}} H_{\lambda} \wedge \mathbf{S},
$$

on the phase space $S^{2} \times S^{2}$. Putting $\hbar_{N, S} \rightarrow 0$ illustrates how the semi-classical limit is related to the limit of adiabatically slow motion. Under the additional assumption $N \gg S$ giving $\hbar_{N} \ll \hbar_{S}$, Hamilton's equations (6) describe the dynamics of an adiabatically coupled system with the motion of $\mathbf{N}$ being much slower than that of $\mathbf{S}$.

\section{Classical description: structure of the generalized moment map}

\subsection{Second integral of motion}

The $S O(2)$ symmetry gives rise to a second integral of motion $J_{z}$, equation (2), which is the projection of the total angular momentum $\mathbf{J}=2 \mathbf{S}+\mathbf{N}$ onto the $z$-axes. Together $H_{\lambda}, J_{z}$ define a one-parameter family of integrable systems with two degrees of freedom.

\subsection{Reduction of symmetry, space of orbits}

The symmetry of the system can be used to reduce the number of degrees of freedom. This is done by mapping each orbit of the $S O(2)$ action on $S^{2} \times S^{2}$ onto the three-dimensional space of orbits. As the group action is not transitive, this is an example of the so-called singular reduction [5] based on the theory of invariants [5, 6, 35].

\footnotetext{
5 Several quantum systems may give rise to the same classical system. See e.g. [30] for an example.
} 


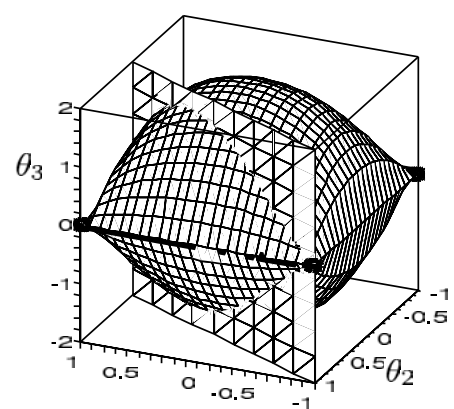

$\theta_{1}$

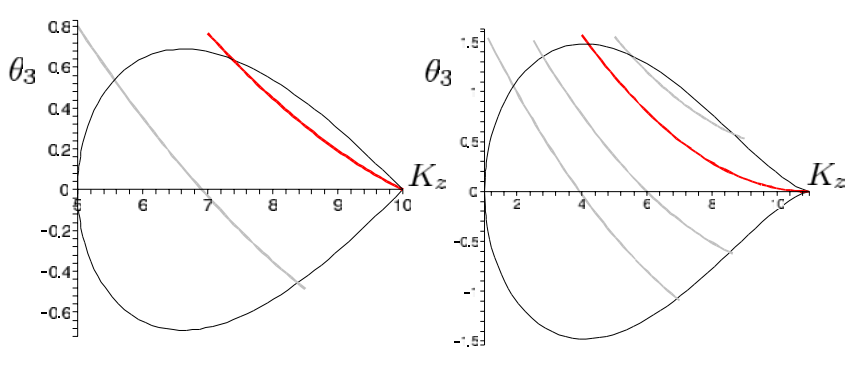

Figure 1. Left: the space of orbits of the $S O(2)$ action. The boundary is given by $\phi=0$. The vertical plane is a section for constant $J_{z}$. Middle: a typical section for $N_{z}=-|\mathbf{N}|,\left|S_{z}\right|<|\mathbf{S}|$. This is a part of the continuous family of singular spaces. The singular orbit at the intersection of the boundary and the constant energy level set has a $\mathbb{Z}_{2}$ stabilizer. Right: a singular section for $J_{z}=2|\mathbf{S}|-|\mathbf{N}|$. The singular orbit situated at the intersection of the constant energy level set and the boundary (critical orbit) has the stabilizer $S O(2)$.

The idea is to see $H_{\lambda}, J_{z}$ as made up of $S O(2)$-invariant polynomials

$$
\begin{aligned}
& \theta_{1}=S_{z}, \quad \theta_{2}=N_{z}, \quad \theta_{3}=N_{-}^{2} S_{+}+N_{+}^{2} S_{-}, \\
& \phi=N_{-}^{2} S_{+}-N_{+}^{2} S_{-},
\end{aligned}
$$

satisfying the algebraic relation [35]

$$
\phi^{2}=\theta_{3}^{2}-4\left(\mathbf{S}^{2}-\theta_{1}^{2}\right)\left(\mathbf{N}^{2}-\theta_{2}^{2}\right)^{2}
$$

An orbit of the $S O(2)$ action (1) can be characterized by the value of the three algebraically independent invariants $\theta_{i}, i=1,2,3$, and the sign of the linearly independent, but algebraically dependent through (7), invariant $\phi$. The space of orbits can then be visualized in a $\left(\theta_{1}, \theta_{2}, \theta_{3}\right)$ coordinate system as a closed body defined by

$$
\theta_{3}^{2}-4\left(\mathbf{S}^{2}-\theta_{1}^{2}\right)\left(\mathbf{N}^{2}-\theta_{2}^{2}\right)^{2} \leqslant 0
$$

The space of orbits is shown in figure 1 (left). Its interior points correspond to two orbits distinguished by the sign of $\phi$ while the boundary points correspond to a single orbit.

There are three equivalence classes of orbits forming different strata in the initial $4 \mathrm{D}$-phase space, which are as follows:

- Generic circular orbits with a trivial stabilizer (4 D regular stratum).

- A continuous family of orbits for $N_{z}= \pm|\mathbf{N}|$ and $\left|S_{z}\right|<|\mathbf{S}|$ with the stabilizer $\mathbb{Z}_{2}$ (2 D critical stratum). These orbits are half as long as a generic orbit.

- Four isolated critical orbits for $\left(S_{z}, N_{z}\right)=( \pm|\mathbf{S}|, \pm|\mathbf{N}|)$ with the stabilizer $S O$ (2) (0 D critical stratum).

\subsection{Generalized moment map}

The most natural way to characterize qualitatively the classical dynamics for an integrable model is to introduce the generalized moment map [4, 36, 37]

$$
\mathbf{F}_{\lambda}=\left(H_{\lambda}, J_{z}\right): S^{2} \times S^{2} \rightarrow \mathbb{R}^{2},
$$



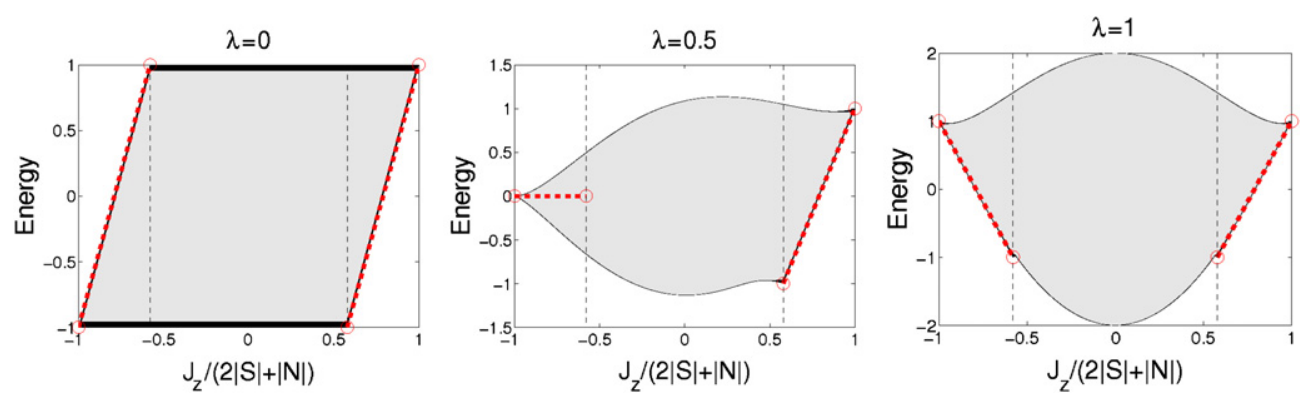

Figure 2. Image $B_{\lambda}$ of the generalized momentum map (9) for different values of the external parameter $\lambda$. For $\lambda \sim 1 / 2$, there are critical values inside $B_{1 / 2}$ and the system has fractional monodromy.

which maps the compact phase space to a bounded domain $B_{\lambda} \subset \mathbb{R}^{2}$ which can be expressed as the union of regular and critical values of (9) $B_{\lambda}=B_{\lambda}^{r} \cup B_{\lambda}^{c}$. Quite naturally, the shape of $B_{\lambda}$ depends on the parameter $\lambda$ as figure 2 shows.

The generalized moment map defines a fibration over $B_{\lambda}$ : for fixed $b \in B_{\lambda}$ the dynamics takes place on the fiber $\mathbf{F}_{\lambda}^{-1}(h, j)$. Here and later on we use $j$ to denote possible values of $J_{z}$.

From the Arnold-Liouville theorem, it is known that the fiber over a regular value $b \in B_{\lambda}^{r}$ is a 2-torus [4] (since $S^{2} \times S^{2}$ is compact). We denote it here as a regular fiber. The critical strata in the phase space are mapped via (9) to the critical values $b_{c} \in B_{\lambda}^{c}$. These critical values can form isolated points inside the image of the generalized moment map, boundary lines or special points on the boundary, and even lines of critical values situated inside the image of the generalized moment map. Critical values which belong to the boundary of the image correspond typically to tori of lower dimension (circles or points). Critical values situated inside the image have nontrivial inverse images $[19,38,39]$.

For $\lambda=\lambda^{*}=1 / 2$ some of the critical values are found in the interior $B_{\lambda^{*}}$, and such values correspond to nontrivial fibers responsible for the appearance of fractional monodromy [13-15].

It is convenient to make a coordinate transformation in the space of orbits

$$
J_{z}=2 S_{z}+N_{z}=2 \theta_{1}+\theta_{2}, \quad K_{z}=S_{z}-2 N_{z}=\theta_{1}-2 \theta_{2},
$$

where $K_{z}$ is the variable varying on $J_{z}$ sections.

Figure 1 shows singular $J_{z}$ sections together with constant level sets of energy. It is easy to see geometrically that in order to have critical values on the image of the energy-momentum map inside the domain of regular values, it is necessary that the energy level going through the singular orbit intersects the boundary of the orbit space at the singular orbit. In other words, we need to compare the slope of the constant energy level at the singular orbit with the slope of two boundary lines of the $J_{z}$-constant section at the singular point on the boundary.

It should be noted that at the critical orbit the geometrical form of the $J_{z}$ section $\pm\left(-2|\mathbf{N}|+|\mathbf{S}|-K_{z}\right)^{3 / 2}$ implies that the two boundary lines form a cusp and have the same zero slope. Due to that, the energy section going through the critical point intersects the boundary only if the energy section has itself the zero slope at the critical orbit and this can happen only for $\lambda=1 / 2$. The typical images of the energy momentum map for $\lambda<1 / 2, \lambda=1 / 2, \lambda\rangle 1 / 2$ are shown in figure 2 . We do not go into details of the evolution of the line of singular values (dashed red line in figure 2) near $\lambda=1 / 2$ which are related to the possible appearance of a second connected component in the inverse image of the generalized moment map. We note that such complications (as compared with a more simple scenario of the appearance of integer 


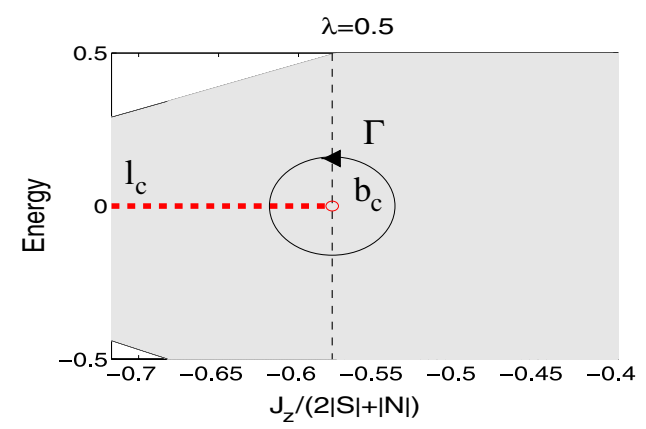

Figure 3. The local setup in the image of the generalized moment map $B_{\lambda^{*}}$ when the system has fractional monodromy. The line $l_{c}$ of critical values is the projection of the critical stratum formed by curled tori $[13,14]$. The critical value $b_{c}$ is the projection of curled pinched torus which is the fiber with the critical point $(0,0,-|\mathbf{N}|),(0,0,|\mathbf{S}|)$. The figure is done for the ratio $J / S=15 / 2$.

monodromy [6] through the Hamiltonian Hopf bifurcation [16]) are only due to the presence of the cusp singularity in the space of orbits. Moreover, it is not essential for the appearance of the line of singular values together with the end point inside the generalized moment image as shown in figure 2 (center) which is responsible for the presence of fractional monodromy.

\subsection{Integer monodromy: holonomy of the lattice bundle}

For each regular value $b \in B_{\lambda}^{r}$, the periodicity of the Arnold-Liouville tori defines a 2 D-lattice $\mathcal{L}_{b}$ isomorphic to the regular lattice $\mathbb{Z}^{2}$ [4]. Over critical values $b_{c} \in B_{\lambda}^{c}$, the fiber is singular and we no longer have a well-defined lattice. To detect the presence of singular fibers, it is sufficient to consider the lattice bundle [5]

$$
\mathcal{L}: \bigcup_{b \in \Gamma} \mathcal{L}_{b} \rightarrow B_{\lambda}^{r}
$$

restricted to a loop $\Gamma:[0,1] \rightarrow B_{\lambda}^{r}$ in $B_{\lambda}^{r}$. This loop passes only through regular values. As $\Gamma(0)=\Gamma(1)$ lifting of $\Gamma$ induces an automorphism on fibers, $\operatorname{Aut}\left(\mathcal{L}_{b=\Gamma(0)}\right) \in S L(2, \mathbb{Z})$. The bundle $\left.\mathcal{L}\right|_{\Gamma}$ depends only on the homotopy type of $\Gamma$ such that we only have to consider equivalence classes of loops (the fundamental group), $\pi_{1}\left(B_{\lambda}^{r}\right)$. The monodromy map is now defined as

$$
\mu: \pi_{1}\left(B_{\lambda}^{r}\right) \rightarrow S L(2, \mathbb{Z}),
$$

which is an example of the holonomy concept $[5,32]^{6}$. Note that here $\mathcal{L}$ is a flat bundle, i.e. its curvature tensor vanishes.

When the system has an isolated critical value, $\pi_{1}\left(B_{\lambda}^{r}\right)=\mathbb{Z}$. The corresponding monodromy map depends on the topology of the singular fiber and results in the transformation of basis cycles of regular tori which can be expressed as a linear combination with integer coefficients. This gives standard integer monodromy [9, 5, 38].

As opposed to numerous known examples of integer monodromy in the literature, we no longer have isolated critical values. This is shown in figure 3 where the critical value

$$
b_{c}=\mathbf{F}_{\lambda}((0,0,|\mathbf{N}|),(0,0,-|\mathbf{S}|))
$$

${ }^{6}$ Holonomy has become a unifying concept in physics, e.g. the Berry phase is seen as the holonomy of a $U$ (1)-bundle $[26,40]$. 
of the generalized moment map is connected to a line, $l_{c}$, of critical values. In such a case we have $\pi_{1}\left(B_{\lambda}^{r}\right)=0$ for every $\lambda$ as is seen from figure 2 , so there is no integer monodromy. However, a suitable restriction of the monodromy map (12) allows one to use closed paths which cross a critical line and surround a critical value $b_{c}$. Transformation of the basis cycles of regular tori after their parallel transfer along such closed paths leads to the new notion of fractional monodromy [13-16].

\subsection{Fractional monodromy: restriction of basis cycles}

To determine the fractional monodromy map, we have to describe how the fibers are continuously modified as we go along the closed path $\Gamma$ in the base space $B_{\lambda}$ of the integrable fibration and how the line of critical values can be crossed using only a subgroup of cycles generating the fibers.

The local setup in $B_{\lambda}$ is sketched in figure 3. In order to understand the evolution of basis cycles of tori along the contour $\Gamma$, we need to note that the trajectories of $J_{z}$ are closed and well defined along all $\Gamma$. They are due to the $S O(2)$ symmetry of the problem and can be used to represent $\gamma_{1}$, the first of the two cycles generating the first homology group of the regular fibers.

The second cycle $\gamma_{2}$ is chosen as the intersection of fibers with an auxiliary plane. Details of this construction are given in figure 7 of [14]. To pass continuously along $\Gamma$ this cycle has to be a double loop. The applicability of the previous discussion of fractional monodromy [14] to the case of the model Hamiltonian (3) studied in the present work is confirmed by reducing the present model $\left(H_{\lambda}, J_{z}\right)$ into the normal form of fractional monodromy presented in [14]. This is done in appendix A.

Due to the splitting of one of the basis cycles when crossing the singular stratum, the monodromy map is only defined for an index 2 subgroup of the first homology group of regular fibers. This is the essence of fractional monodromy. The relation between the initial basis cycles $\gamma_{1,2}$ and the final basis cycles $\gamma_{1,2}^{\prime}$, at the end of the cyclic evolution along $\Gamma$, can be written in the matrix form as [13]

$$
\left(\begin{array}{c}
\gamma_{1}^{\prime} \\
2 \gamma_{2}^{\prime}
\end{array}\right)=\underbrace{\left(\begin{array}{cc}
1 & 0 \\
-1 & 1
\end{array}\right)}_{\mu_{c l}}\left(\begin{array}{c}
\gamma_{1} \\
2 \gamma_{2}
\end{array}\right) .
$$

A formal extension of the monodromy map to the basis of the whole homology group of regular fibers introduces fractional coefficients and a monodromy matrix

$$
\mu_{c l}=\left(\begin{array}{cc}
1 & 0 \\
-1 / 2 & 1
\end{array}\right) \in S L(2, \mathbb{Q}) .
$$

This implies that the preimage $\mathbf{F}_{\lambda^{*}}^{-1}(\Gamma)$ does not factorize as $\mathbb{T}^{2} \times S^{1}$ and hence the momentum map is not a principal $\mathbb{T}^{2}$-fiber bundle [5]. There is then no unique way of labeling tori in a vicinity of the pinched curled torus, and no global set of action-angle coordinates can be introduced.

\section{Quantum monodromy}

The Einstein-Brillouin-Kramer (EBK) quantization introduces quantum numbers by picking out a set of regular tori [31]

$$
\int_{\gamma_{k}} p \mathrm{~d} q=2 \pi \hbar\left(n_{k}+\alpha_{k} / 4\right), \quad k=1,2,
$$



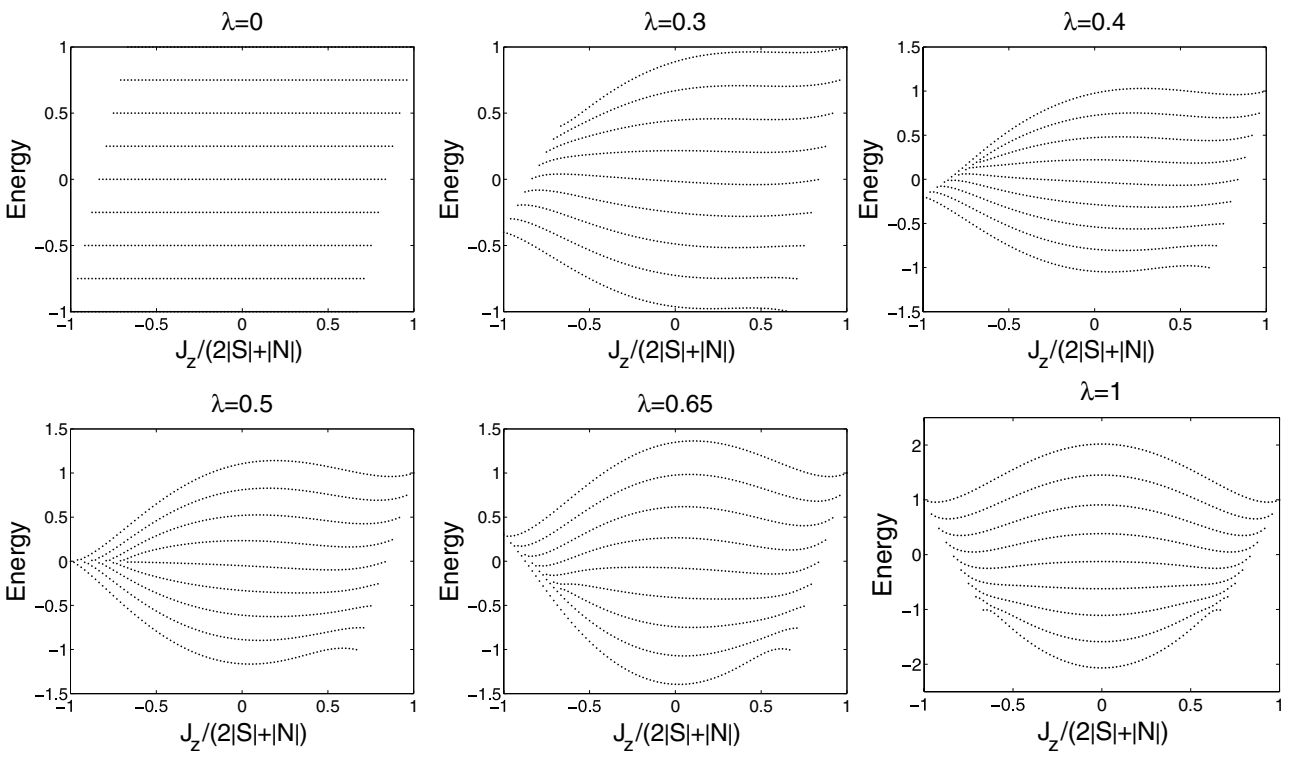

Figure 4. Modifications of the joint spectrum as $\lambda$ varies, $\lambda=0 \rightarrow 1$. The bands are labeled $\sigma=-S, \ldots, S$ from the bottom up. Here $S=4, N=40$. For $\lambda=1 / 2$ there is fractional quantum monodromy due to the presence of the line of critical values inside of the image of the moment map. As $1 / 2>\lambda \rightarrow \lambda>1 / 2$ there is a modification of the band structure due to the displacement of the line of critical values from the upper side of the boundary of the moment image towards the lower side, passing through the interior (see figure 2). For $\lambda=0$, there are $\mathcal{N}=2 S+1$ states on each line, whereas for $\lambda=1$, there are $\mathcal{N}_{\sigma}=(2 S+1)+4 \sigma$ states on the line $\sigma$, as is explained below.

where $\gamma_{k}$ are basis cycles, generators of the tori, and $\alpha_{k}$ are Maslov indices. Given this, it is no surprise that classical monodromy manifests itself in quantum systems as quantum monodromy. The existence of this property was first demonstrated on the quantum spherical pendulum [41] and later defined as the dual of classical monodromy [18] $]^{7}$.

The EBK rules lead to a 2 D-lattice of quantum states - or joint spectrum-in $B_{\lambda}$. From (16) the distance between consecutive quantum states decreases as $\hbar \rightarrow 0$. Our model is a coupling of two angular momenta $\mathbf{S}$ and $\mathbf{N}$ with effective Planck constants $\hbar_{S}$ and $\hbar_{N}$, respectively. The assumption $S \ll N$ leads to $\hbar_{N} \ll \hbar_{S}$ and to the existence of two scales in the joint spectrum. This explains the local band structure easily observed in figure 4 . We label the bands by the quantum number of $S_{z}, \sigma=-S, \ldots, S$.

For $\lambda=0$, the joint spectrum forms globally a regular lattice which possesses a well defined (up to a similarity transformation with the $S L(2, Z)$ matrix) elementary cell over the whole lattice. This means that there exists a global labeling of states. The lattice remains to be regular (just in a slightly deformed form) for the $\lambda$-dependent family of integrable systems up to $\lambda \sim 1 / 2$. At $\lambda=1 / 2$ the presence of a one-dimensional defect is clearly seen within the regular part of the lattice. This defect results in a modification of the bands. For $\lambda=1$ we again have a globally regular lattice but now with a different band structure.

For $\lambda \sim 1 / 2$, the effect of the defect on the lattice is characterized (up to conjugation) by an element $\mu_{q m}$ determined in the following way (see figure 5):

7 This is only strictly true in the semi-classical limit. In such a case, the distance between consecutive points in the spectrum goes to zero and we recover a continuous description. 

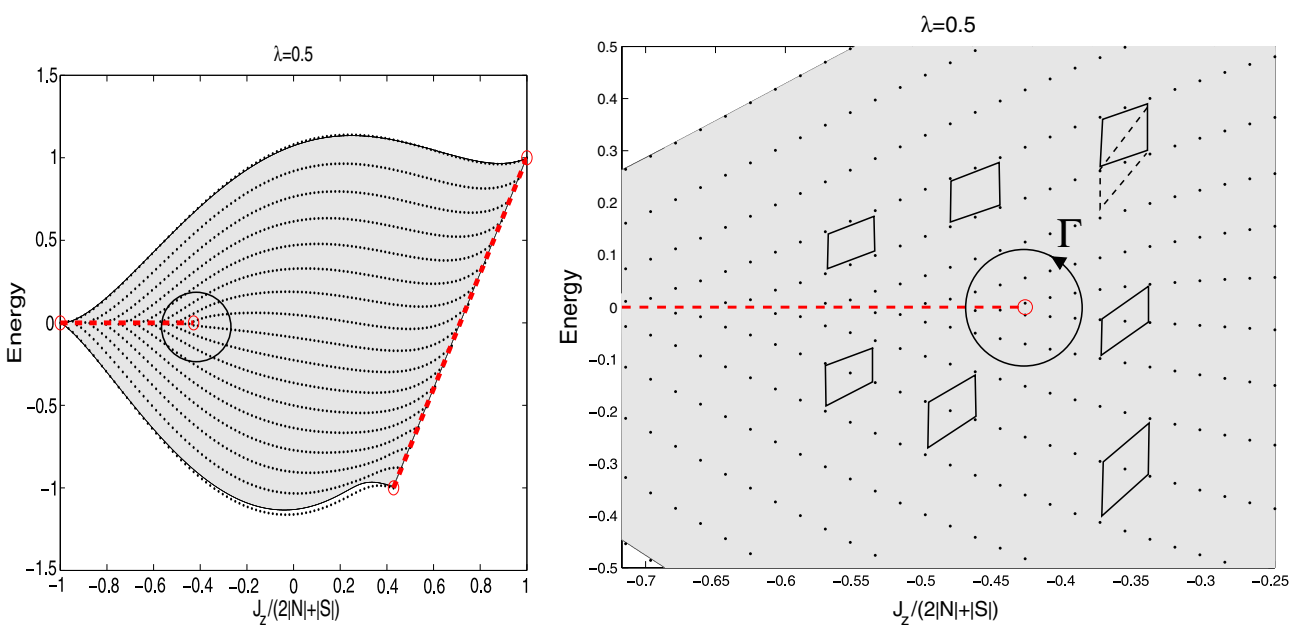

Figure 5. Joint spectrum for $S=8, N=40$ and $\lambda=1 / 2$ which shows the effect of fractional monodromy. Left: the global view of the joint spectrum. Right: parallel transport of the double cell along a closed path crossing once the line of critical values and surrounding the critical value $\left(J_{z}=2 S-N, E=0\right)$ of the generalized moment map. For $S=8, N=40$ we have $J_{z} /(2|S|+|N|)=-3 / 7 \approx-0.4286$.

- Make a choice of cell. To pass the line defect the cell should be doubled in the $J_{z}$ direction. This is the quantum analogue of the restriction imposed on the choice of passable cycles in section 3.5. Cell doubling is not necessary in the case of integer monodromy [6].

- Moving along the path $\Gamma$ between initial and final points, the elementary cell does not change as long as the path remains within the class of homotopically trivial paths. However, after translation along a path $\Gamma$ as shown in figure 5 we return with a different cell. A rescaling as done in section 3.5 gives

$$
\mu_{\mathrm{qm}}=\left(\begin{array}{cc}
1 & 1 / 2 \\
0 & 1
\end{array}\right) \in S L(2, \mathbb{Q})
$$

which is the quantum monodromy matrix (after a formal rescaling of the cells) ${ }^{8}$.

The nontriviality of monodromy shows that no unique set of quantum numbers exists which can be used to label states in the joint spectrum [42]. This is of special importance for molecular physics where effective quantum numbers are typically introduced on the basis of experimental spectral information using extrapolation within effective models.

\subsection{Decomposition into sub-lattices}

Let $j$ label the eigenvalues of $J_{z}$, the second integral of motion, nd $\mathcal{N}_{j}$ be the dimension of the associated eigenspace, i.e. the number of states with $J_{z}=$ const in figure 4 . The number of states function (figure 6) is a quasi-polynomial, i.e. polynomial in $j$ with coefficients being periodic in $j$ :

$$
\mathcal{N}_{j}= \begin{cases}2 S+1, & |j| \leqslant N-2 S \\ \frac{1}{2}\left(J-|j|+\frac{1}{2}\left(3+(-1)^{J+|j|}\right)\right), & \text { otherwise. }\end{cases}
$$

${ }^{8}$ Here we observe the duality between classical and quantum monodromy explicitly as $\mu_{\mathrm{qm}}={ }^{t} \mu_{\mathrm{cl}}^{-1}[18]$. 


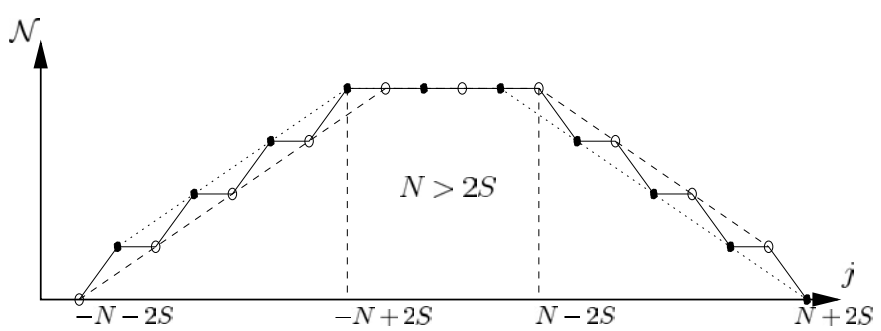

Figure 6. The number-of-states function $\mathcal{N}_{j}$ is a quasi-polynomial (full line). The existence of two length scales in the system is due to the 1:2 diagonal $S O(2)$-action. Retaining only the linear term, i.e. restricting to either even (o) or odd (•) values of $j$, results in two subsystems with integer monodromy.
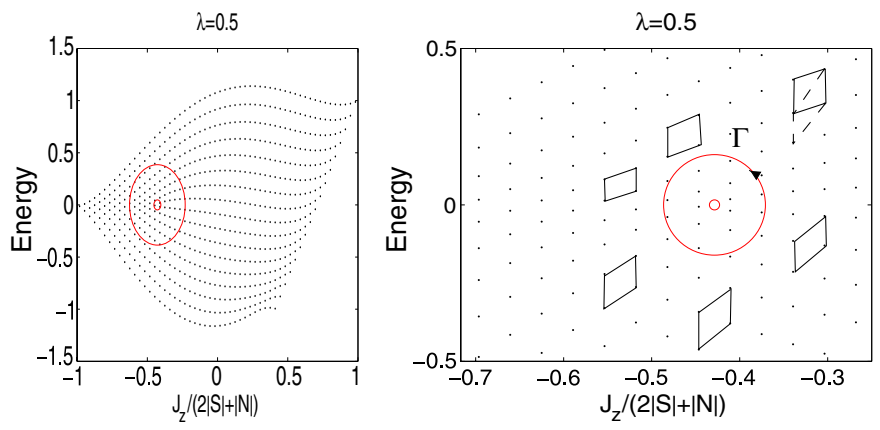

Figure 7. Index 2 sub-lattice of the joint spectrum for odd values of $J_{z}$. This sub-ladtticed possesses only one isolated critical value. The path encircling this critical value is characterized by integer monodromy. The situation is similar for even $J_{z}$.

This reflects the existence of two different scales in the system. A large-scale behavior is associated with the polynomial part, whereas a small-scale behavior is characterized by the oscillating term. This is a direct consequence of the 1:2 diagonal $S O(2)$ action as described in section 2.1 .

Restricting ourselves to only even or odd values of $J_{z}$ amounts to ignoring the oscillating part of (18). This gives integer monodromy on each index 2 sub-lattice of the joint spectrum as is shown in figure 7. Disregarding the small-scale behavior, our system reduces to two systems with 1:(-1) resonance of the type found in [6].

Integer monodromy on index 2 sub-lattices should be compared with the monodromy matrix (14), i.e. before the formal rescaling of the restricted basis cycle. This is another way of showing how fractional monodromy can be seen as integer monodromy for an appropriate subset of basis cycles.

\subsection{Quantum monodromy and redistribution of states}

Returning to figure 4, we observe that the breaking of the band structure is related to the appearance of monodromy and to a rearrangement of bands seen as a transfer of states from the lower to the upper bands. Counting the number of states before and after a modification of the position of the singular stratum on the image of the generalized moment map gives

$$
\Delta \mathcal{N}_{\sigma}=4 \sigma, \quad \sigma=-S, \ldots, S,
$$

where $\mathcal{N}_{\sigma}$ is the number of states in the $\sigma$ th band (labeled from the bottom up). 


\section{Semi-quantum description: Chern index}

In this section we give a different interpretation of the redistribution of states, observed in figure 4. This will be done with a topological interpretation of the number of states within each band. For that purpose, we will derive and explain the index formula (35).

We now proceed to consider the semi-quantum description which is valid in the limit $S \ll N$, with no assumption on $S$. Here the slow motion of $\mathbf{N}$ is classical, and for any given value of $\mathbf{N}$ the fast motion of $\mathbf{S}$ is quantum mechanical and depends on $\mathbf{N}$ : the fast motion is generated by the Hamiltonian $\hat{H}_{\mathbf{N}, \lambda}$ acting in $\mathcal{H}_{S}$ and obtained by substituting the operators $\hat{\mathbf{N}}$ by the classical variable $\mathbf{N} \in S^{2}$.

This operator has normalized eigenstates

$$
\hat{H}_{\lambda, \mathbf{N}}\left|\psi_{\sigma}(\lambda, \mathbf{N})\right\rangle=E_{\sigma}(\lambda, \mathbf{N})\left|\psi_{\sigma}(\lambda, \mathbf{N})\right\rangle
$$

with $\sigma=-S \ldots+S$. The eigenvalues, $E_{\sigma}(\lambda, \mathbf{N}): S^{2} \rightarrow \mathbb{R}$, seen as functions of $\mathbf{N}$ form $2 S+1$ bands calculated in the following way.

The quantum Hamiltonian is an operator-valued symbol defined by

$$
\left\langle\mathbf{N}\left|\hat{H}_{\lambda}\right| \mathbf{N}\right\rangle=\hat{H}_{\lambda, \mathbf{N}}+O(1 / N),
$$

and explicitly given by

$$
\begin{aligned}
& \mathbf{N} \in S^{2} \mapsto \hat{H}_{\lambda, \mathbf{N}}=\mathbf{K}_{\lambda}(\mathbf{N}) \cdot \frac{\hat{\mathbf{S}}}{|\mathbf{S}|}, \\
& \mathbf{K}_{\lambda}(\mathbf{N})=\left(\frac{2 \lambda}{|\mathbf{N}|^{2}}\left(N_{+}^{2}+N_{-}^{2}\right), \frac{-2 i \lambda}{|\mathbf{N}|^{2}}\left(N_{+}^{2}-N_{-}^{2}\right),(1-\lambda)+\frac{\lambda}{|\mathbf{N}|} N_{z}\right),
\end{aligned}
$$

the principal symbol with respect to $\mathbf{N}$.

Explicit eigenvectors are constructed from the angular momentum basis vectors by applying the rotation taking the $z$-axis into $\mathbf{K}_{\lambda}$

$$
\left|\psi_{\sigma}(\lambda, \mathbf{N})\right\rangle=\mathrm{e}^{\mathbf{K}_{\lambda}(\mathbf{N}) \cdot \hat{\mathbf{s}}}|\sigma\rangle, \quad \sigma=-S, \ldots, S,
$$

and the spectrum is

$$
\begin{aligned}
E_{\sigma}(\lambda, \mathbf{N}) & =\frac{\sigma}{|\mathbf{S}|}\left|\mathbf{K}_{\lambda}(\mathbf{N})\right| \\
& =\frac{\sigma}{|\mathbf{S}|} \sqrt{\left(\frac{4 \lambda}{|\mathbf{N}|^{2}}\right)^{2}\left(N_{x}^{2}+N_{y}^{2}\right)^{2}+\left((1-\lambda)+\frac{\lambda}{|\mathbf{N}|} N_{z}\right)^{2}},
\end{aligned}
$$

which shows that the only degeneracy between bands occurs for

$$
\begin{aligned}
& N_{x}=N_{y}=0 \Rightarrow N_{z}= \pm|\mathbf{N}|, \\
& (1-\lambda)+\lambda \frac{N_{z}}{|\mathbf{N}|}=0,
\end{aligned}
$$

with solution $\left(\lambda^{*}, \mathbf{N}^{*}\right)=(1 / 2,(0,0,-|\mathbf{N}|))$. In this case, there is a collective degeneracy between all bands in the semi-quantum spectrum due to the high degree of symmetry of the model $[1,6]^{9}$.

$9 k$ th-order eigenvalue degeneracies of a Hermitian operator occur in a space of dimension $\left(\operatorname{dim}_{\text {parameters }}-\left(k^{2}-1\right)\right)$ [43]. With three independent parameters $(\lambda, \mathbf{N}) \in[0,1] \times S^{2}$ only point-wise degeneracies between pairs of eigenvalues are generic, i.e. cannot be removed by perturbing the model. The important point is that with three parameters we shall always have band degeneracies where the Chern index can be 'exchanged' [12]. 


\subsection{Complex line bundles over $S^{2}$}

For each $\sigma=-S, \ldots, S$ there is a natural vector bundle structure associated with a parameterdependent operator constructed as follows.

The normalized eigenvectors (23) are only defined up to a phase factor but the projector

$$
\hat{P}_{\sigma}: \mathbf{N} \in S^{2} \mapsto\left|\psi_{\sigma}(\lambda, \mathbf{N})\right\rangle\left\langle\psi_{\sigma}(\lambda, \mathbf{N})\right|
$$

onto the corresponding eigenspace is well defined and associates with each point $\mathbf{N} \in S^{2}$ a one-dimensional complex subspace of $\mathcal{H}_{S}$. This defines $2 S+1$ complex line bundles $L_{s} \rightarrow S^{2}$ for almost all values of $\lambda$ (except when the degeneracy mentioned in the previous section is encountered). Each bundle has an isomorphism class depending on $\lambda \in[0,1]$ and characterized by a single integer $C_{\sigma} \in \mathbb{Z}$, the so-called Chern index $[12,44]$.

\subsection{Trivial topology}

For $\lambda=0$ eigenstates form the usual angular momentum basis set $\left|\psi_{\sigma}(0, \mathbf{N})\right\rangle=|\sigma\rangle$. As these states are parameter independent, we have $2 S+1$ trivial line bundles over $S^{2}$ characterized by $C_{\sigma}=0$. As the topology remains unchanged under continuous deformations, this remains true until the sphere spanned by $\mathbf{N}$ encounters $\left(\lambda^{*}, \mathbf{N}^{*}\right)$ at the south pole.

This happens for $\lambda=1 / 2$ and the collective degeneracy can be seen as a trivial rank $_{\mathbb{C}} 2 S+1$ bundle over $S^{2}$. In fact, since the total space $\mathcal{H}_{S}$ is a trivial vector bundle

$$
C=\sum_{\sigma=-S}^{S} C_{\sigma}=0,
$$

for all values of $\lambda$.

\subsection{Nontrivial topology}

As the only degeneracy occurs at $\left(\lambda^{*}, \mathbf{N}^{*}\right)$, it is sufficient to calculate $C_{\sigma}^{\prime}$ for $\lambda=1$. This is done algebraically by defining the Chern index $C_{n}^{\prime}$ as a sum of oriented zeros of a global section $[12]^{10}$.

A choice of a reference coherent state $\left|\mathbf{S}_{0}\right\rangle$ defines a global section

$$
\hat{P}_{\sigma}(\mathbf{N})\left|\mathbf{N}_{0}\right\rangle=\left|\psi_{\sigma}(1, \mathbf{N})\right\rangle\left\langle\psi_{\sigma}(1, \mathbf{N}) \mid \mathbf{S}_{0}\right\rangle,
$$

where $\hat{P}_{\sigma}(\mathbf{N})$ is the projector onto the $\sigma$ th eigenspace in $\mathcal{H}_{S}$ spanned by $\left|\psi_{\sigma}(1, \mathbf{N})\right\rangle$. The section has the same zeros as the Husimi distribution

$$
\mathcal{H}_{\sigma}(\mathbf{S})=\left|\left\langle\psi_{\sigma}(1, \mathbf{N}) \mid \mathbf{N}_{0}\right\rangle\right|^{2},
$$

of $\left|\mathbf{S}_{0}\right\rangle$. Here $\left|\psi_{\sigma}\right\rangle$ is simply a rotation of the angular momentum eigenstates $|\sigma\rangle$ with a Husimi distribution known to have $(S-\sigma)$ oriented zeros at $\mathbf{K}_{1}(\mathbf{N})$ and $-(S+\sigma)$ oriented zeros at $-\mathbf{K}_{1}(\mathbf{N})$ [33].

Introducing spherical coordinates $(\Phi, \Theta)$ on the parameter sphere

$$
\mathbf{K}_{1}(\Phi, \Theta)=\left(4 \sin ^{2}(\Theta) \cos (2 \Phi), 4 \sin ^{2}(\Theta) \sin (2 \Phi), \cos (\Theta)\right),
$$

we see that as $(\Phi, \Theta)$ cover the sphere once $\left|\psi_{\sigma}\right\rangle$ cover the phase space twice. Then each set of zeros pass over all points on the sphere-including $\mathbf{S}_{0}$ - twice and

$$
C_{\sigma}^{\prime}=2(S-\sigma+(-(S+\sigma)))=-4 \sigma .
$$

${ }^{10}$ A section is a continuous choice of elements in each fiber. A non-vanishing section globally defines a frame and hence a global separation of the bundle. In this case the bundle is trivial: it is isomorphic to $S^{2} \times \mathbb{C}$. 
The change in the Chern index for the $\sigma$ th bundle is then

$$
\Delta C_{\sigma}=C_{\sigma}^{\prime}-C_{\sigma}=-4 \sigma,
$$

as $\lambda=0 \rightarrow 1$.

\subsection{Exchange of states and indices: an index formula}

In section 4.2 the change in the number of states was found to be $\Delta \mathcal{N}_{\sigma}=4 \sigma$ such that

$$
\Delta C_{\sigma}=-\Delta \mathcal{N}
$$

and $\mathcal{N}_{\sigma}+C_{\sigma}$ is conserved for all values of $\lambda$. When $\lambda=0$ we have $\hat{H}_{0}(\mathbf{N})=\hat{S}_{z}$ and $\mathcal{N}_{\sigma}=2 S+1=\operatorname{dim} \mathcal{H}_{N}$ which leads to

$$
\mathcal{N}_{\sigma}=\operatorname{dim} \mathcal{H}_{S}-C_{\sigma}=(2 S+1)+4 \sigma,
$$

relating the topology of a complex line bundle in the semi-quantum description to the number of quantum states in a band [7]. This so-called index formula on the sphere is the simplest case of the Atiyah-Singer index formula [45].

\section{Discussion}

Quantum systems with a slow-fast coupled motion are very common in nature, the textbook example being that of a rovibrational molecular system $[1,3,6,7,22,30]$. We have given a model example of such a system with a specific (1:2 diagonal) action of the dynamical symmetry group which has the additional property of being integrable.

The raison d'etre of our model is a $S O(2)$ with 1:2 diagonal action leading to fractional monodromy, the essence being a restriction of the monodromy map to an index 2 subset of basis cycles. To our knowledge, this is currently the only example of fractional monodromy in a system with compact phase space. This gives a bounded spectrum which is important when we turn to the physically relevant question of redistribution. The hydrogen atom in the presence of electric and magnetic fields leads under certain conditions to effective models which manifest the fractional monodromy effect [46].

Here we observe that the appearance of monodromy is related to a breaking of the band structure in the joint spectrum. Furthermore, this is associated with a rearrangement of bands seen as a redistribution of quantum states.

In the semi-quantum description the notion of integrability is not present but the redistribution of states appears as a change in the Chern index of the associated complex line bundles. This is the result of a simple index formula expressing the redistribution of levels in terms of Chern indices [7].

Also a recent generalization of the so-called moment polytopes of Atiyah, GuilleminSternberg and Delzant to problems with integer monodromy [11] makes the precise relation between redistribution (Chern index) and general $p / q$-monodromy a pertinent question. Our model can easily be generalized to $1 / k$-monodromy [29] but for the time being the more actual question is to find a physical example of a system exhibiting fractional monodromy and the redistribution phenomenon.

\section{Acknowledgments}

MSH would like to thank LPMMC exquisite hospitality during his masters thesis $(2003 / 04)$. This work was partly supported by the EU project Mechanics and Symmetry in Europe (MASIE), contract no HPRN-CT-2000-00113. 


\section{Appendix. Local structure of the generalized moment map: monodromy}

To establish the presence of fractional monodromy $H_{1 / 2}, J_{z}$ is reduced to a normal form for fractional monodromy presented in [13]. This is done by linearizing around $\left(\mathbf{N}^{*}, \mathbf{S}^{*}\right)=$ $((0,0,|\mathbf{N}|),(0,0,-|\mathbf{S}|))$

$$
\begin{aligned}
& N_{x}=p_{1}, \quad N_{y}=q_{1}, \quad N_{z}=\sqrt{1-\left(N_{x}^{2}+N_{y}^{2}\right)} \simeq 1-\frac{1}{2}\left(p_{1}^{2}+q_{1}^{2}\right), \\
& S_{x}=p_{2}, \quad S_{y}=q_{2}, \quad S_{z}=-\sqrt{1-\left(S_{x}^{2}+S_{y}^{2}\right)} \simeq-1+\frac{1}{2}\left(p_{2}^{2}+q_{2}^{2}\right),
\end{aligned}
$$

where $\left(q_{1}, p_{1}, q_{2}, p_{2}\right) \in T_{\mathbf{N}^{*}} S^{2} \times T_{\mathbf{S}^{*}} S^{2} \cong \mathbb{R}^{2} \times \mathbb{R}^{2}$ is a set of local symplectic coordinates. Then

$H_{1 / 2}(q, p)=\underbrace{\operatorname{Re}\left[\mathrm{i}\left(q_{1}-\mathrm{i} p_{1}\right)\left(q_{2}-\mathrm{i} p_{2}\right)^{2}\right]}_{H_{0}}+\underbrace{\frac{1}{2}\left(p_{2}^{2}+q_{2}^{2}\right)}_{H_{r}}-\underbrace{\frac{1}{2}\left(p_{1}^{2}+q_{1}^{2}\right)\left(p_{2}^{2}+q_{2}^{2}\right)}_{H_{c}}$,

$J_{z}(q, p)=-\left(p_{1}^{2}+q_{1}^{2}\right)+\frac{1}{2}\left(p_{2}^{2}+q_{2}^{2}\right)$

To find the position of the critical values, we solve

$$
D H_{1 / 2}(q, p)=0, \quad D J_{z}(q, p)=0,
$$

including terms up to third order $\left(q_{i}, p_{i} \ll 1\right)$. There is a corank-2 critical value at $\left(H, J_{z}\right)=(0,0)$ and a line of corank-1 critical points

$$
\left(H, J_{z}\right)=\left(0,-p_{1}^{2}-q_{1}^{2}\right)
$$

in accordance with figure 3 . As $H_{r} 1$ gonly depends on $q_{2}, p_{2}$, it has no influence on the qualitative picture and can be disregarded.

$J_{z}$ is the Hamiltonian of a pair of oscillators in $1:(-2)$ resonance. Together with $H_{0}$, it is the system of functions in involution used to demonstrate the existence of fractional monodromy in [13].

This completes the reduction to the normal form [13, 14].

\section{References}

[1] Pavlov-Verevkin V B, Sadovskií D A and Zhilinskií B I 1988 On the dynamical meaning of diabolic points Europhys. Lett. 6 573-8

[2] Efstathiou K, Sadovskií D A and Zhilinskiì B I 2004 Analysis of rotation-vibration relative equilibria on the example of a tetrahedral four atom molecule SIAM J. Appl. Dyn. Syst. (SIADS) 3 261-351

[3] Cushman R H and Sadovskií D A 2000 Monodromy in the hydrogen atom in crossed fields Physica D 65 166-96

[4] Arnol'd V I 1989 Mathematical Methods of Classical Mechanics (Heidelberg: Springer)

[5] Cushman R H and Bates L M 1997 Global Aspects of Classical Integrable Systems (Basle: Birkhäuser)

[6] Sadovskií D A and Zhilinskiì B I 1999 Monodromy, diabolic points, and angular momentum coupling Phys. Lett. A $256235-44$

[7] Faure F and Zhilinskií B I 2000 Topological Chern indices in molecular spectra Phys. Rev. Lett. 85 960-3

[8] Grondin L, Sadovskií D A and Zhilinskií B I 2002 Monodromy in systems with coupled angular momenta and rearrangement of bands in quantum spectra Phys. Rev. A 65 012105-1-15

[9] Duistermaat J J 1980 On global action angle coordinates Commun. Pure Appl. Math. 33 687-706

[10] Duistermaat J J 1998 The monodromy of the Hamiltonian Hopf bifurcation Z. Angew. Math. Phys. 49 156-61

[11] Ngọc S Vũ 2007 Moment polytopes for symplectic manifolds with monodromy Adv. Math. 208 909-34

[12] Faure F and Zhilinskií B I 2001 Topological properties of the Born-Oppenheimer approximation and implications for the exact spectrum Lett. Math. Phys. 55 219-38

[13] Nekhoroshev N N, Sadovskií D A and Zhilinskií B I 2002 Fractional monodromy of resonant classical and quantum oscillators C. R. Acad. Sci., Paris I 335 985-8 
[14] Nekhoroshev N N, Sadovskií B I and Zhilinskií B I 2006 Fractional Hamiltonian monodromy Ann. Henri Poincaré 7 1099-211

[15] Efstathiou K, Cushman R H and Sadovskií D A 2007 Fractional monodromy in the 1 : (-2) resonance Adv. Math. 209 241-73

[16] Efstathiou K 2005 Metamorphoses of Hamiltonian Systems with Symmetries (Lectures Notes in Mathematics vol 1864) (New York: Springer-Verlag) pp 149

[17] Broer H, Cushman R H and Fassó F 2007 Geometry of KAM tori for nearly integrable Hamiltonian systems Ergod. Th Dyn. Sys. 27 725-41 (Preprint math.DS/0210043)

[18] Ngọc S Vũ 1999 Quantum monodromy in integrable systems Commun. Math. Phys. 203 465-79

[19] Zung T Z 1996 Symplectic topology of integrable Hamiltonian systems: I. Arnol'd-Liouville with singularities Comput. Math. 101 179-215

[20] Zung T Z 2003 Symplectic topology of integrable Hamiltonian systems: II. topological classification Comput. Math. 138 125-56

[21] Cushman R H, Dullin H R, Giacobbe A, Joyeux M, Lynch P, Sadovskií D A and Zhilinskií B I 2004 CO molecule as a quantum realization of the $1: 1: 2$ resonant swing-spring with monodromy Phys. Rev. Lett. 9324302

[22] Joyeux M, Sadovskií D A and Tennyson J 2003 Monodromy of the LiNC/NCLi molecule Chem. Phys. Lett. $382439-42$

[23] Sadovskií D A and Zhilinskiì B I 2006 Quantum monodromy, its generalizations and molecular manifestations Mol. Phys. 104 2595-615

[24] Child M S 2007 Quantum monodromy and molecular spectroscopy Adv. Chem. Phys. at press

[25] Sadovskií D A and Zhilinskiì B I 2007 Hamiltonian systems with detuned 1: $1: 2$ resonance. manifestations of bidromy Ann. Phys., (NY) 232 164-200

[26] Berry M V 1984 Quantal phase factors accompanying adiabatic change Proc. R. Soc. Lond. A $39245-57$

[27] Panati G, Spohn H and Teufel S 2003 Space-adiabatic perturbation theory Adv. Theor. Math. Phys. 7 145-204

[28] Faure F and Zhilinskií B I 2002 Topologically coupled energy bands Phys. Lett. A 302 242-52

[29] Hansen M S 2004 Adiabatically coupled systems: Redistribution, monodromy, and Chern index Master's Thesis Technical University of Denmark

[30] Zhilinskií B I 2001 Symmetry, invariants, and topology in molecular models Phys. Rep. 341 85-171

[31] Landau L and Lifshitz E 1965 Quantum Mechanics (Theo. Phys. vol 3) (Moscow: Mir)

[32] Nakahara M 1990 Geometry, Topology and Physics (Bristol: Hilger)

[33] Lebœuf P 1991 Phase space approach to quantum dynamics J. Phys. A: Math. Gen. 24 4574-86

[34] Zhang W, Feng D H and Gilmore G 1990 Coherent states: theory and some applications Rev. Mod. Phys. 62 867-927

[35] Michel L and Zhilinskií B I 2001 Symmetry, invariants, topology. Basic tools Phys. Rep. 341 11-84

[36] Guillemin V 1994 Moment Maps and Combinatorial Invariants of Hamiltonian $T^{n}$-spaces (Boston: Birkhäuser)

[37] Marsden J E and Ratiu T S 1999 Introduction to Mechanics and Symmetry 2nd ed (Heidelberg: Springer)

[38] Zung T Z 1997 A note on focus-focus singularities Differ. Geom. Appl. 7 123-30

[39] Bolsinov A V and Fomenko A T 1997 Integrable Hamiltonian Systems. Geometry, Topology, Classification (London/Boca Raton, FL: Chapman and Hall/CRC Press)

[40] Simon B 1983 Holonomy, the quantum adiabatic theorem, and Berry's phase Phys. Rev. Lett. 51 2167-70

[41] Cushman R H and Duistermaat J J 1988 The quantum mechanical spherical pendulum Bull. Am. Soc. 19 475-9

[42] Giacobbe A, Cushman R H, Sadovskií D A and Zhilinskií B I 2004 Monodromy of the quantum 1:1:2 resonant swing spring J. Math. Phys. 45 5076-100

[43] Avron J, Zur B and Raveh A 1988 Adiabatic transport in multiply connected systems Rev. Mod. Phys 60 873-915

[44] Griffiths P and Harris J 1978 Principles of Algebraic Geometry (New York: Wiley)

[45] Fedosov F 2000 The Atiyah-Bott-Patodi method in deformation quantizaton Commun. Math. Phys. 209 691-728

[46] Efstathiou K, Sadovskií D A and Zhilinskií B I 2007 Classification of perturbations of the hydrogen atom by small static electric and magnetic fields Proc. R. Soc. Lond. A $\mathbf{4 6 3}$ 1771-90 